\title{
Qu'est-ce que la tolérance? Perspectives sur Voltaire, sous la direction de Jurgen Siess
}

\section{Nadia Minerva}

\section{(2) OpenEdition}

1 Journals

\section{Edizione digitale}

URL: https://journals.openedition.org/studifrancesi/39428

DOI: 10.4000/studifrancesi.39428

ISSN: 2421-5856

\section{Editore}

Rosenberg \& Sellier

\section{Edizione cartacea}

Data di pubblicazione: 1 décembre 2004

Paginazione: 375-376

ISSN: 0039-2944

\section{Notizia bibliografica digitale}

Nadia Minerva, «Qu'est-ce que la tolérance? Perspectives sur Voltaire, sous la direction de Jurgen Siess», Studi Francesi [Online], 143 (XLVIII | II) | 2004, online dal 30 novembre 2015, consultato il 19 mai 2021 URL: http://journals.openedition.org/studifrancesi/39428 ; DOI: https://doi.org/10.4000/studifrancesi. 39428

Questo documento è stato generato automaticamente il 19 mai 2021.

\section{(c) $(1) \ominus$}

Studi Francesi è distribuita con Licenza Creative Commons Attribuzione - Non commerciale - Non opere derivate 4.0 Internazionale. 


\title{
Qu'est-ce que la tolérance? Perspectives sur Voltaire, sous la direction de Jurgen Siess
}

\author{
Nadia Minerva
}

\section{NOTIZIA}

Qu'est-ce que la tolérance? Perspectives sur Voltaire, sous la direction de Jurgen SIEsS, Ferney-Voltaire, Centre Intemational d'Étude du XVIIIe siècle, 2002, pp. 174.

1 Com'è noto, il tema della tolleranza è da tempo una costante degli studi voltairiani, studi che hanno visto nel Patriarca di Ferney il portavoce più autorevole del secolo, non per l'originalità, ma per l'impatto sull'opinione pubblica, la capacità cioè di elaborare una sintesi efficace ed incisiva in grado di dare risonanza alle idee dei suoi predecessori. Rispetto alla vasta bibliografia critica sull'argomento, il presente volume sceglie la prospettiva dell'attualità - possibile? auspicabile? - del pensiero e delle strategie settecentesche, relativamente ad un tema la cui drammatica evidenza continua a sollevare, all'alba del terzo millennio, giustificate preoccupazioni.

2 Il titolo del volume riprende il celebre quesito posto da Voltaire nel Dictionnaire philosophique, quesito nel quale il curatore del volume vede un interrogativo che attende ancora una risposta. L'intolleranza del nostro tempo indurrebbe ad evocare le lotte di Voltaire e ad assumerne le posizioni. Ma, precisa Siess, i mutamenti del quadro socio-storico, del pensiero politico ed etico sono tali rispetto al Siècle des Lumières che occorre innanzitutto chiedersi cosa abbia in comune la nozione di tolleranza degli uomini dei Lumi con la nostra, cosa sia ancora condivisibile oggi delle interrogazioni e delle lotte scaturite allora. E soprattutto come ripensare nel terzo millennio il "paradosso della tolleranza»? Come essere tolleranti di fronte all'intolleranza? Quanto si deve essere intolleranti per realizzare una società tollerante? Per questa riflessione, Siess ha convocato ad un convegno intemazionale svoltasi a Gerusalemme nel 2000 
(luogo e data simbolici) studiosi francesi ed israeliani per uno stimolante dialogo interculturale e interdisciplinare dove si sono confrontati storici, filosofi, studiosi della letteratura, specialisti dell'analisi del discorso.

L'opera raccoglie dodici studi in quattro sezioni «cronologiche» (dal dibattito in seno al pensiero protestante che prende origine da Locke fino a quello odierno che ha visto l'impegno - tra gli altri - di storici e filosofi come Baczko e Ricœur): «Histoire des religions, histoire des idéologies»; «Voltaire, en contexte»; «Voltaire: discours et argumentation»; «Perspectives actuelles sur Voltaire et la tolérance».

Nella prima parte, Michael HEYD (Origines chrétiennes de la tolérance: le point de vue protestant, pp. 11-21), Elhanan YAKIRA (La tolérance entre la "Theodicée» et «Candide», pp. 23-33) e Myriam YARDENI (Paix perpétuelle et tolérance des religions: le projet de l'abbé de Saint-Pierre,pp. 35-43) situano Voltaire rispetto alla storia dell'idea di tolleranza e al pensiero dell'alterità Nella seconda e terza parte, Dominique BouReL (Voltaire, les Juifs et l'Europe, pp. 47-57), Roland MORTIER (La tolérance selon Voltaire: entre l'irénisme et la combativité, pp. 59-65), Isabelle MARTIN (L'animal, l'humain, l'inhumain dans le «Traité sur la tolérance», pp. 67-74), Carole DORNIER (Critique de l' autorité et autorité de la critique dans le "Traité sur la tolérance» pp. 77-86), André MAGNAN ("Ces véritables voix humaines...»: Voltaire en histrion d'une tolérance théiste, pp. 87-101), Michele ВОВОВКА-КАНАN («Impartial mais sensible»: l'ethos dans le "Traité sur la tolérance», pp. 103-116), e Christian PLANTIN (La construction de l'évidence dans le "Traité sur la tolérance», pp. 117-137), propongono analisi degli aspetti storici e discorsivi della complessa «scène d'énonciation» del Traité sur la tolérance. In particolare, gli ultimi due contributi, redatti per l'opera a stampa, aprono una nuova prospettiva rispetto al convegno del 2000, studiando l'argomentazione voltairiana. Retorica ed argomentazione che, secondo Plantin, conferiscono al Traité «évidence et luminosité». Tuttavia, conclude rassegnato Plantin, «il n'appartieni pas aux seules puissances du discours de déterminer le destin» del dibattito plurisecolare sulla tolleranza (p. 137).

5 Roland Mortier, come il curatore di questi atti, si assume il compito di stabilire dei limiti quando, ricordando le due diverse accezioni del termine «tolleranza» (assenza di divieti e di imposizioni che limitano le libertà individuali, oppure comprensione ed acccttazione della differenza), invita a non cadere nell'anacronismo di esigere da Voltaire e dal Settecento la piena consapevolezza e l'impegno per la realizzazione di entrambe le idee di tolleranza. La seconda non poteva che costituire un orizzonte d'attesa ancora lontano per i philosophes (p. 59).

Nell'ultima parte, Jürgen sIEss (La place de Voltaire dans le débat contemporain sur la tolérance, pp. 141-152 e David HEYD, Pédagogie de la tolérance: problèmes et possibilités de solution. pp. 153-162) riflettono sul valore e sul paradosso dell'idea di tolleranza del Patriarca in una società multiculturale come la nostra. Siess rileva l'interesse odierno del pensiero di Voltaire in tre sfere, quelle dei pensieri liberale e repubblicano che animano il Settecento per sfociare nel dibattito rivoluzionario sui diritti dell'uomo e quella dei rapporti tra tolleranza e impegno dell'intellettuale. Il critico giunge però a conclusioni moderatamente critiche: «sans doute la pensée de Voltaire sur la tolérance ne répond pas exactement aux besoins complexes de nos sociétés démocratiques et multiculturelles. Mais dans ses insuffisances autant que dans son apport, elle continue à alimenter une réflexion sur la tolérance dont les enjeux sont cruciaux» (p. 152). Si potrebbe commentare che Voltaire ha raggiunto in parte il suo obiettivo: svegliare le coscienze e tener viva la discussione. Sulla stessa linea, si pone l'esito dell'indagine di 
Heyd, il quale evidenzia il contributo di Voltaire alla storia dello sviluppo della moderna tolleranza: «le premier pas» è stato compiuto da Voltaire là dove aspira ad istituire una specie di coesistenza passiva «fondée sur la prise de conscience pratique du prix que coûte un conflit perpétue!» (p. 161). Un primo passo al quale dovrebbe tuttavia seguire una fase più matura - e dunque consona allo scenario dei giorni nostri - nella quale si possano stabilire rapporti stabili tra gruppi conflittuali, rapporti fondati sul rispetto reciproco.

7 Se si vogliono scorgere nel volume degli schieramenti, si potrebbe opporre alle posizioni di Siess e di Heyd la voce di due appassionati e al tempo stesso lucidi settecentisti come Bronislaw Baczko e Roland Mortier. Il primo può proclamare - in un passaggio tratto da Job, mon ami: promesses du bonheur et fatalité du mal del 1997, assunto ad esergo dell' Introduction di Siess - la sua adesione alla strategia voltairiana di lotta all'intolleranza riassunta nel motto "Écraser l'infame». Il secondo, pur rilevando «faiblesses» e «outrances» di Voltaire, vede nel Traité e nella battaglia contro l'intolleranza condotta infaticabilmente dal Patriarca, «l'honneur de sa vie». Una battaglia «qui garde tout son sens à une époque comme la nôtre où nous voyons revivre les sectes, les intégrismes, les fatwas, les crimes légitimés par la foi et par le dogme religieux mais aussi politique» (p. 65).

8 Chiude il volume una traduzione in ebraico della celebre Prière à Dieu di Voltaire che dobbiamo a Arza APFELROIT. 